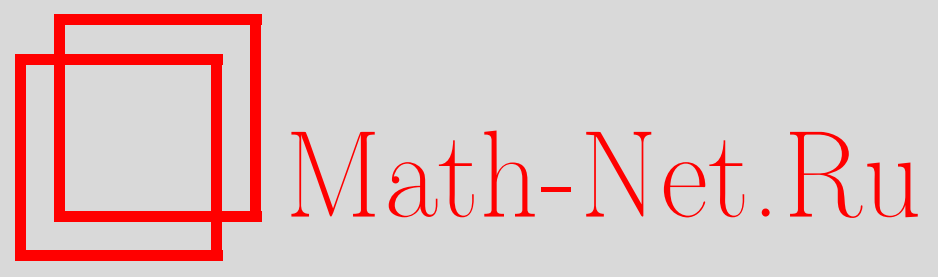

А. В. Косточка, В. Д. Мазуров, Л. Я. Савельев, Число
q-ичных слов с ограничениями на длину максимальной
серии, Дискрет. матем., 1998, том 10, выпуск 1, 10-19
DOI: https://doi.org/10.4213/dm413

Использование Общероссийского математического портала Math-Net.Ru подразумевает, что вы прочитали и согласны с пользовательским соглашением http://www . mathnet.ru/rus/agreement

Параметры загрузки:

IP: 44.207 .124 .84

26 апреля 2023 г., 14:27:17

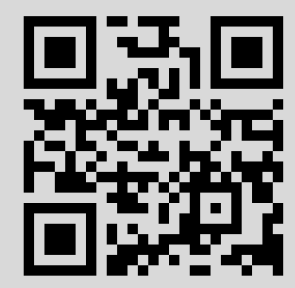




\section{Число $q$-ичных слов с ограничениями на длину максимальной серии}

(C) 1998 г. А. В. Косточка, В. Д. Мазуров, Л. Я. Савельев

Установлено, что число $g(q, s, n)$ слов длины $n$ в $q$-буквенном алфавите таких, что длина любого подслова из стоящих рядом одинаковых букв не превосходит $s$, весьма близка к $\lambda^{n}$, где $\lambda$ - наибольший деиствительный корень полинома $x^{s+1}-q x^{s}+q-1$. Нанддено предствление $\lambda$ в виде суммы ряда. Результаты позволяют вычислять асимптотические значения $g(q, s, n)$ и функции $h(q, s, n)=g(q, s, n)-g(q, s-1, n)$ при $n \rightarrow \infty$ и $s>c \log n$ для любого фиксированного $c>0$.

Работа выполнена при поддержке Российского фонда фундаментальных исследований, проекты 96-01-01614, 96-01-01893 и 96-01-01496 соответственно для каждого из авторов.

\section{Введение}

Пусть $G(q, s, n)$ обозначает множество слов длины $n$ в алфавите $\{0,1, \ldots, q-1\}$, в которых длина любой серии (т.е. подслова из стоящих рядом одинаковых букв) не превосходит $s$, а $H(q, s, n)$ обозначает подмножество слов из $G(q, s, n)$, содержащих хотя бы одну серию длины $s$. Пусть, далее, $g(q, s, n)=|G(q, s, n)|, h(q, s, n)=$ $|H(q, s, n)|$.

Задача подсчета $g(q, s, n)$ и $h(q, s, n)$ связана с рядом других постановок. В частности, $g(2, s ; n)$ (соответственно, $h(2, s, n)$ ) равно удвоенному числу представлений числа $n$ в виде суммы упорядоченных слагаемых, каждое из которых не превосходит $s$ (соответственно, максимальное из которых равно $s$ ). Задача подсчета $g(q, s, n)$ является частным случаем задачи вычисления вероятности того, что в случайном $q$-ичном слове длины $n$ длина максимальной серии не превосходит $s$. В. Л. Гончаров [4] вывел общую производящую функцию для ряда вероятностных задач, связанных с максимальными сериями для $q=2$, когда каждая из букв слова независимо от других с вероятностью $p$ принимает одно из значений и с вероятностью $1-p$ другое. Используя эту формулу, он нашел асимптотику распределения вероятностей длины максимальной серии в типичных случаях. Позднее Л. Я. Савельевым $[9,14]$ и другими исследовались аналогичные задачи при более сложных зависимостях событий; некоторые ссылки можно найти в $[9,6]$. А. Д. Коршунов [6] нашел асимптотику $h(2, s, n)$ при $n \rightarrow \infty$ и $s \geqslant(1 / 2) \log _{2} n+2 \log _{2} \log _{2} n$. В настоящей работе используется подход, отличный от подхода в [6], и получены более общие результаты. 
Понятно, что

$$
h(q, s, n)=g(q, s, n)-g(q, s-1, n)
$$

$\boldsymbol{K}$

$$
g(q, s, n)=q^{n}, \quad n=1,2, \ldots, s .
$$

Задача нахождения $g(q, s, n)$ сводится к исследованию корней некоторых многочленов. Пусть

$$
\begin{aligned}
& A_{q, s}(x)=x^{s}-(q-1)\left(1+x+x^{2}+\ldots+x^{s-1}\right), \\
& B_{q, s}(x)=(x-1) A_{q, s}(x)=x^{s+1}-q x^{s}+q-1 .
\end{aligned}
$$

Основным результатом настоящей работы является следующая теорема.

Теорема 1. Пусть $q \geqslant 2, s \geqslant 2$ - натуралънъе иисла, $\lambda_{1}=\lambda_{1}(q, s)$ - наиболъиий положстельний корень многочлена $A_{q, s}(x)$, a

$$
b(q, s)=\frac{q\left(\lambda_{1}-1\right)}{(q-1)\left((s+1) \lambda_{1}-s q\right)} .
$$

Тогда при $n \geqslant s$ число $g(2, s, n)$ равно ближайшему $x b(2, s) \lambda_{1}^{n}$ четному числу, $a$ для $q \geqslant 3$ число $g(q, s, n)$ равно блиюайшему $\propto b(q, s) \lambda_{1}^{n}$ челому числу.

Из дальнейшего будет видно, что $b(q, s)$ близко к 1.

Следствие 1. $\Pi p u n \geqslant s$

$$
h(2, s, n)=2\left(\left\lfloor 0.5 b(2, s) \lambda_{1}(2, s)^{n}\right\rceil-\left\lfloor 0.5 b(2, s-1) \lambda_{1}(2, s-1)^{n}\right\rceil\right),
$$

$u \partial \Omega \Omega q \geqslant 3$

$$
h(q, s, n)=\left\lfloor b(q, s) \lambda_{1}(q, s)^{n}\right\rceil-\left\lfloor b(q, s-1) \lambda_{1}(q, s-1)^{n}\right\rceil,
$$

где $\lfloor z\rceil$ обозначает ближайиее $\boldsymbol{x} z$ челое число.

Таким образом, задача нахождения или оценивания $g(q, s, n)$ и $h(q, s, n)$ эквивалентна задаче нахождения или оценивания $\lambda_{1}(q, s)$. Понятно, что

$$
\lambda_{1}(q, 2)=(q-1)(1+\sqrt{5}) / 2
$$

и $g(2,2, n)$ равно удвоенному $n$-му числу Фибоначчи.

Формулы для нахождения $\lambda_{1}(q, s)$ дает следующий результат.

Теорема 2. При $s \geqslant 2 \partial \Omega я \lambda_{1}(q, s)$ справедливы формулы

$$
\begin{aligned}
& \lambda_{1}(q, s)=q\left(1-\sum_{k=1}^{\infty} \frac{1}{k}\left(\begin{array}{c}
k(s+1)-2 \\
k-1
\end{array}\right)\left(\frac{q-1}{q^{s+1}}\right)^{k}\right), \\
& \lambda_{1}(q, s)=q \exp \left\{-\sum_{k=1}^{\infty} \frac{1}{k}\left(\begin{array}{c}
k(s+1)-1 \\
k-1
\end{array}\right)\left(\frac{q-1}{q^{s+1}}\right)^{k}\right\} .
\end{aligned}
$$

Работа построена следующим образом. В параграфе 2 выведены свойства корней вспомогательных многочленов. На основе этого в параграфе 3 доказана теорема 1. В параграфе 4 доказана теорема 2. В параграфе 5 выводятся асимптотические своиства $g(q, s, n)$ и $h(q, s, n)$ при $n \rightarrow \infty$. Параграф 6 посвящен обсуждению полученных результатов. 


\section{1. О корнях многочлена $A_{q, s}(x)$}

Предложение 1. Пусть $q \geqslant 2, s \geqslant 2$ - натуральнье иисла. Тогда многочлен $A_{q, s}(x)$ не имеет кратных корней. Он обладает вещественным корнем $\lambda_{1}$, принадлежсиим промежутку $\left(q-q^{1-s}, q\right)$. Модули всех остальных корней меньие единичь.

Доказательство. Производная многочлена $B_{q, s}(x)$ имеет ровно два корня: 0 (кратности $s-1)$ и $\alpha=q s /(s+1)$. Очевидно, 0 не является корнем $B_{q, s}(x)$. Предположим, что $\alpha$-- корень $B_{q, s}(x)$. Поскольку все коэффициенты $B_{q, s}(x)$ являются целыми и старший коэффициент равен 1 , любой рациональный корень $B_{q, s}(x)$ обязан быть целым (см., например, $[7, \S 57])$ и являться делителем $q-1$. Так как $s$ и $s+1$ взаимно просты, $s+1$ делит $q$ и $\alpha=q_{0} s$, где $q_{0}=\alpha / s$ - целое число. Поскольку $q_{0}$ делит $q$ и $q-1$, необходимо $q_{0}=1$ и $\alpha=s=q-1$. Следовательно,

$$
0=B_{q, s}(\alpha)=s^{s+1}-(s+1) s^{s}+s=-s^{s}+s,
$$

что невозможно.

Итак, $B_{q, s}(x)$ не имеет общих корней с производной, то есть не имеет кратных корней. Следовательно, $A_{q, s}(x)=B_{q, s}(x) /(x-1)$ тоже не имеет кратных корней.

Заметим, что

$$
\begin{aligned}
B_{q, s}\left(q-q^{1-s}\right) & =\left(q-q^{1-s}\right)^{s}\left(q-q^{1-s}-q\right)+q-1 \\
& =-q\left(1-q^{-s}\right)^{s}+q-1 \\
& <-q\left(1-s q^{-s}\right)+q-1 \\
& =s q^{-s}-1<0 \\
B_{q, s}(q) & =q^{s+1}-q^{s+1}+q-1=q-1>0 .
\end{aligned}
$$

Следовательно, $A_{q, s}(x)$ имеет некоторый корень $\lambda_{1}$ в промежутке $(q-1, q)$.

Допустим, что $A_{q, s}(x)$ имеет корень $\lambda \neq \lambda_{1}$ с $|\lambda|>1$. Поскольку $\lambda$ и $\lambda_{1}-$ корни $B_{q, s}(x)$,

$$
q-\lambda=\frac{q-1}{\lambda^{s}}, \quad q-\lambda_{1}=\frac{q-1}{\lambda_{1}^{s}}
$$

Отсюда

$$
\lambda-\lambda_{1}=\frac{q-1}{\lambda_{1}^{s}}-\frac{q-1}{\lambda^{s}}=(q-1) \frac{\lambda^{s}-\lambda_{1}^{s}}{\lambda^{s} \lambda_{1}^{s}},
$$

и после деления на $\lambda-\lambda_{1}$ получаем, что

$$
1=(q-1) \frac{\lambda_{1}^{s-1}+\lambda_{1}^{s-2} \lambda+\ldots+\lambda^{s-1}}{\lambda^{s} \lambda_{1}^{s}} .
$$

Переходя к модулям, находим, что

$$
\begin{aligned}
1 & \leqslant(q-1) \frac{\lambda_{1}^{s-1}+\lambda_{1}^{s-2}|\lambda|+\ldots+\left|\lambda^{s-1}\right|}{|\lambda|^{s} \lambda_{1}^{s}} \\
& <(q-1) \frac{\lambda_{1}^{s-1}+\lambda_{1}^{s-2}+\ldots+\lambda_{1}+1}{\lambda_{1}^{s}}
\end{aligned}
$$

поскольку $|\lambda|>1$. Так как $\lambda_{1}-$ корень $A_{q, s}(x)$, последняя дробь равна 1 . Полученное противоречие завершает доказательство предложения. 
Лемма 1. Для каждого отличного от $\lambda_{1}$ корня $\lambda$ многочлена $B_{q, s}(x)$ справедливо неравенство

$$
\frac{|1-\lambda|}{|q-\lambda|^{2}} \leqslant \frac{1}{2 \sqrt{q}(q-1)}
$$

Доказателъство. Ввиду предложения 1 достаточно доказать, что неравенство (8) справедливо для любого комплексного $\lambda$, принадлежащего кругу $|\lambda| \leqslant 1$.

Итак, пусть $|\lambda| \leqslant 1, \lambda=a+b i$, где $a$ и $b-$ действительные числа. Пусть $d=|1-\lambda|, D=|q-\lambda|$. Тогда

$$
\begin{aligned}
d^{2} & =1-2 a+a^{2}+b^{2} \\
D^{2} & =q^{2}-2 q a+a^{2}+b^{2}=q^{2}-2(q-1) a+d^{2}-1 .
\end{aligned}
$$

Поэтому на дуге окружности $|1-\lambda|=d$, заключенной внутри круга $|\lambda| \leqslant 1$, минимум $D$ достигается при наибольшем $a$, го есть на пересечении с окружностью $|\lambda|=1$. Поэтому в дальнейшем полагаем $a^{2}+b^{2}=1$. Тогда, согласно (9), $2 a=2-d^{2}$ и

$$
\frac{d}{D^{2}}=\frac{d}{q^{2}+d^{2}-1-(q-1)\left(2-d^{2}\right)}=\frac{d}{(q-1)^{2}+q d^{2}} .
$$

Ясно, что максимум последнего выражения для положительных $d$ достигается при $d=(q-1) / \sqrt{q}$ и равен $1 /(2 \sqrt{q}(q-1))$. Лемма доказана.

\section{2. Доказательство теоремы 1}

Пусть $G(i, q, s, n)$ - подмножество слов из $G(q, s, n)$, первая буква которых есть $i$, $i=0,1 \ldots, q-1$, a $G(j, i, q, s, n)$ - подмножество слов из $G(i, q, s, n)$, первые $j$ букв которых суть $i, i=0,1 \ldots, q-1, j=1 \ldots, s$. Нужные нам уравнения выводятся из следующих простых тождеств.

$$
\begin{array}{rlrl}
|G(i, q, s, n)| & =|G(k, q, s, n)| & & \forall i, k=0,1 \ldots, q-1 ; \\
|G(q, s, n)| & =q|G(i, q, s, n)| & & \\
& =q \sum_{j=1}^{s}|G(j, i, q, s, n)| & \\
|G(j, i, q, s, n)| & =\left|\bigcup_{k \neq i} G(k, q, s, n-j)\right| \\
& =(q-1)|G(i, q, s, n-j)| \\
& =\frac{q-1}{q}|G(q, s, n-j)| .
\end{array}
$$

Согласно (11) и (12), для каждого $n \geqslant s+1$

$$
g(q, s, n)=(q-1)(g(q, s, n-1)+g(q, s, n-2)+\ldots+g(q, s, n-s)) .
$$


Таким образом, при фиксированных $q$ и $s$ последовательность $g(q, s, n)$ является возвратной последовательностью, определяемой уравнениями (13) и начальными значениями (2). Характеристическим многочленом (см. [8, 3, 13]) последовательности $g(q, s, n)$ является $A_{q, s}(x)$. По предложению 1 все корни многочлена $A_{q, s}(x)-$ простые. Следовательно (см., например, $[8,3,13])$, решение уравнений (13) имеет вид

$$
g(q, s, n)=b_{1} \lambda_{1}^{n}+\ldots+b_{s} \lambda_{s}^{n}
$$

где $\lambda_{1}, \ldots, \lambda_{s}$ суть корни $A_{q, s}(x)$. Чтобы определить коэффициенты $b_{1}, \ldots, b_{s}$, воспользуемся (2). Получаем систему уравнений

$$
\begin{array}{r}
q=\lambda_{1} b_{1}+\lambda_{2} b_{2}+\ldots+\lambda_{s} b_{s}, \\
q^{2}=\lambda_{1}^{2} b_{1}+\lambda_{2}^{2} b_{2}+\ldots+\lambda_{s}^{2} b_{s}, \\
\ldots \ldots \ldots \ldots \ldots \ldots \ldots \ldots \ldots \ldots \ldots \ldots \ldots \\
q^{s}=\lambda_{1}^{s} b_{1}+\lambda_{2}^{s} b_{2}+\ldots+\lambda_{s}^{s} b_{s} .
\end{array}
$$

По правилу Крамера $b_{i}=D_{i} / D, i=1, \ldots, n$, где

$$
D=\left|\begin{array}{cccc}
\lambda_{1} & \lambda_{2} & \ldots & \lambda_{s} \\
\lambda_{1}^{2} & \lambda_{2}^{2} & \ldots & \lambda_{s}^{2} \\
\ldots \ldots & \ldots \ldots & \ldots \\
\lambda_{1}^{s} & \lambda_{2}^{s} & \ldots & \lambda_{s}^{s}
\end{array}\right|
$$

а $D_{i}$ получается из $D$ заменой $i$-го столбца на столбец свободных коэффициентов системы (15). Имеем

$$
\begin{aligned}
D & =\lambda_{1} \lambda_{2} \ldots \lambda_{s} W\left(\lambda_{1}, \lambda_{2}, \ldots, \lambda_{s}\right) \\
D_{i} & =\lambda_{1} \ldots \lambda_{i-1} q \lambda_{i+1} \ldots \lambda_{s} W\left(\lambda_{1}, \ldots, \lambda_{i-1}, q, \lambda_{i+1}, \ldots, \lambda_{s}\right)
\end{aligned}
$$

где $W\left(z_{1}, \ldots, z_{s}\right)$ обозначает определитель Вандермонда от переменных $z_{1}, \ldots, z_{s}$. Так как

$$
W\left(z_{1}, \ldots, z_{s}\right)=\prod_{i<j}\left(z_{j}-z_{i}\right)
$$

получаем, что

$$
\frac{D_{i}}{D}=\frac{q}{\lambda_{i}} \frac{\prod_{j \neq i}\left(q-\lambda_{j}\right)}{\prod_{j \neq i}\left(\lambda_{i}-\lambda_{j}\right)}
$$

Поскольку $\lambda_{1}, \lambda_{2}, \ldots, \lambda_{s}-$ корни $A_{q, s}(x)$,

$$
\prod_{j \neq i}\left(q-\lambda_{j}\right)=\frac{A_{q, s}(q)}{q-\lambda_{i}}=\frac{1}{q-\lambda_{i}}
$$


Так как $A_{q, s}\left(\lambda_{i}\right)=B_{q, s}\left(\lambda_{i}\right)=0$, находим, что

$$
\begin{aligned}
\prod_{j \neq i}\left(\lambda_{i}-\lambda_{j}\right) & =\lim _{x \rightarrow \lambda_{i}} \frac{A_{q, s}(x)}{x-\lambda_{i}} \\
& =\lim _{x \rightarrow \lambda_{i}} \frac{B_{q, s}(x)}{(x-1)\left(x-\lambda_{i}\right)} \\
& =\lim _{x \rightarrow \lambda_{i}} \frac{1}{x-1} \lim _{x \rightarrow \lambda_{i}} \frac{B_{q, s}(x)-B_{q, s}\left(\lambda_{i}\right)}{x-\lambda_{i}} \\
& =\frac{1}{\lambda_{i}-1}\left[B_{q, s}(x)\right]_{x=\lambda_{i}}^{\prime} \\
& =\frac{(s+1) \lambda_{i}^{s}-s q \lambda_{i}^{s-1}}{\lambda_{i}-1} .
\end{aligned}
$$

Помня, что $\lambda_{i}^{s}\left(q-\lambda_{i}\right)=q-1$, получаем, что

$$
\frac{D_{1}}{D}=\frac{q}{\lambda_{1}} \frac{1}{q-\lambda_{1}} \frac{\lambda_{1}-1}{\lambda_{1}^{s-1}\left((s+1) \lambda_{1}-s q\right)}=\frac{q\left(\lambda_{1}-1\right)}{(q-1)\left((s+1) \lambda_{1}-s q\right)} .
$$

Так как $\left|\lambda_{i}\right|<1$ для $i \geqslant 2$, при этих $i$ и $n \geqslant s$ справедлива оценка

$$
\left|b_{i} \lambda_{i}^{n}\right|=\left|\lambda_{i}^{n} D_{i} / D\right| \leqslant \frac{\left|\lambda_{i}\right|^{n-s} q\left|1-\lambda_{i}\right|}{\left|q-\lambda_{i}\right|\left|-(s+1) \lambda_{i}+s q\right|} .
$$

Заметим, что

$$
\left|-(s+1) \lambda_{i}+s q\right|=\left|s\left(q-\lambda_{i}\right)-\lambda_{i}\right| \geqslant s\left|q-\lambda_{i}\right|-1>(s-1)\left|q-\lambda_{i}\right| .
$$

Учитывая лемму 1 , находим, что при $i \geqslant 2$ и $n \geqslant s$

$$
\left|b_{i} \lambda_{i}^{n}\right| \leqslant \frac{\left|\lambda_{i}\right|^{n-s} q\left|1-\lambda_{i}\right|}{(s-1)\left|q-\lambda_{i}\right|^{2}} \leqslant \frac{q}{2 \sqrt{q}(q-1)(s-1)} .
$$

Отсюда

$$
\left|g(q, s, n)-b_{1} \lambda_{1}^{n}\right| \leqslant(s-1) \frac{q}{2 \sqrt{q}(q-1)(s-1)}=\frac{\sqrt{q}}{2(q-1)} .
$$

Так как $q-1>\sqrt{q}$ при $q \geqslant 3$, утверждение теоремы для $q \geqslant 3$ доказано. Поскольку число слов в $G(2, s, n)$, начинающихся с 0 и с 1 , одинаково, $g(2, s, n)$ эетуо. Тәким образом, из (16) вытекает справедливость теоремы.

\section{3. Формулы для $\lambda_{1}(q, s)$}

Для вычисления $\lambda_{1}(q, s)$ удобно использовать теорему Лагранжа об обращении рядов (см., например, [12, п.452]).

Теорема 3. Пусть $f(y) u \phi(y)$ - функции от $y$, аналитические в некоторой окрестности точки $a, u$ я яяяется решением уравнения

$$
y=a+x \phi(y)
$$


Тогда $f$ может быть разложена в ряд по формуле

$$
f(y)=f(a)+\sum_{k=1}^{\infty} \frac{x^{k}}{k !} \frac{d^{k-1}}{d y^{k-1}}\left[f^{\prime}(y) \phi(y)^{k}\right]_{y=a} .
$$

Понятно, что число $\lambda$ является корнем $B_{q, s}(x)$, если и только если $\mu=q / \lambda$ является корнем уравнения

$$
y=1+\frac{q-1}{q^{s+1}} y^{s+1} .
$$

Уравнение (19) является частным случаем уравнения (17) с $a=1, x=$ $(q-1) / q^{s+1}, \phi(y)=y^{s+1}$. Применив теорему 3 к (19) для $f(y)=q / y$ и заметив, что

$$
\begin{gathered}
f^{\prime}(y) \phi(y)^{k}=-\frac{q}{y^{2}} y^{k(s+1)}=-q y^{k(s+1)-2} \\
\frac{1}{k !} \frac{d^{k-1}}{d y^{k-1}}\left[f^{\prime}(y) \phi(y)^{k}\right]_{y=a}=-\frac{q}{k}\left(\begin{array}{c}
k(s+1)-2 \\
k-1
\end{array}\right) y^{k s-1},
\end{gathered}
$$

получаем для близкого к 1 корня $\mu$ уравнения (19) формулу

$$
q / \mu=q-q \sum_{k=1}^{\infty} \frac{1}{k}\left(\begin{array}{c}
k(s+1)-2 \\
k-1
\end{array}\right)\left(\frac{q-1}{q^{s+1}}\right)^{k} .
$$

По признаку Коши этот ряд сходится при любых $s \geqslant 2, q \geqslant 2$. Из формулы вкдно, что $q-1<q / \mu<q$. Как отмечено выше, $q / \mu$ является корнем $B_{q, s}(x)$. Значит, $q / \mu=\lambda_{1}(q, s)$, и формула (6) доказана.

Аналогично, для вывода формулы (7) достаточно применить теорему 3 к (19) для $f(y)=\ln (q / y)($ cp. [11, с. 187]). Получим, что

$$
\ln \lambda_{1}(q, s)=\ln q-\sum_{k=1}^{\infty} \frac{1}{k}\left(\begin{array}{c}
k(s+1)-1 \\
k-1
\end{array}\right)\left(\frac{q-1}{q^{s+1}}\right)^{k}
$$

\section{4. Об асимптотике $g(q, s, n)$ и $h(q, s, n)$}

В качестве приближений для $\lambda_{1}$ можно брать конечные суммы из (6). Введем обозначения

$$
\begin{aligned}
\varphi(x) & =\frac{q(x-1)}{(q-1)((s+1) x-s q)} x^{n} \\
a_{k} & =\frac{1}{k}\left(\begin{array}{c}
k(s+1)-2 \\
k-1
\end{array}\right) \\
z_{m} & =z_{m}(q, s)=q\left(1-\sum_{k=1}^{m} a_{k}\left(\frac{q-1}{q^{s+1}}\right)^{k}\right) .
\end{aligned}
$$

Теорема 1 утверждает, что $\varphi\left(\lambda_{1}(q, s)\right)$ почти совпадает с $g(q, s, n)$. Оценим погрешность аппрокгимации выражения $\varphi\left(\lambda_{1}(q, s)\right)$ с помощью выражения $\varphi\left(z_{m}^{n}\right)$. Поскольку

$$
a_{k} \leqslant \frac{(k s+k / 2)^{k-1}}{k !} \leqslant \frac{(e(s+1 / 2))^{k}}{k(s+1 / 2)}
$$


справедлива оценка

$$
z_{m}-\lambda_{1}=q \sum_{k=m+1}^{\infty} a_{k}\left(\frac{q-1}{q^{s+1}}\right)^{k} \leqslant q\left(\frac{e(s+1 / 2)(q-1)}{q^{s+1}}\right)^{m+1}
$$

Напомним, что $\lambda_{1}>q-q^{1-s}$ по предложению 1 . Нетрудно проверить, что при $6 \leqslant s \leqslant n-1$ и $x>q-q^{1-s}$ функция $\varphi(x)$ возрастает, а дробь

$$
\frac{q(x-1)}{(q-1)((s+1) x-s q)}=\frac{q}{(q-1)(s+1)} \frac{x-1}{x-s q /(s+1)}
$$

убывает с ростом $x$. Следовательно, поскольку $z_{m}>\lambda_{1}$,

$$
\begin{aligned}
1>\frac{\varphi\left(\lambda_{1}\right)}{\varphi\left(z_{m}\right)} \geqslant \frac{\lambda_{1}^{n}}{z_{m}^{n}} & =\left(1-\frac{z_{m}-\lambda_{1}}{z_{m}}\right)^{n} \\
& \geqslant 1-n \frac{z_{m}-\lambda_{1}}{z_{m}} \\
& \geqslant 1-2 n\left(\frac{e(s+1 / 2)(q-1)}{q^{s+1}}\right)^{m+1} .
\end{aligned}
$$

Так как при любых $q \geqslant 2, s \geqslant 4$ справедливо неравенство

$$
e(s+1 / 2)(q-1) q^{-s-1}<1 / 2,
$$

при таких $q, s$ и $m>2 \log _{2} n$

$$
1>\frac{\varphi\left(\lambda_{1}\right)}{\varphi\left(z_{m}\right)}>1-2 n 2^{-1-2 \log _{2} n}>1-1 / n \text {. }
$$

При $n \rightarrow \infty$ и $s \geqslant 0,5 \log _{q} n+2 \log _{q} \log _{q} n$ значение $\varphi\left(z_{1}\right)$ асимптотически совпадает с $g(q, s, n)$. Действительно, в этом случае

$$
\begin{aligned}
1>\frac{\varphi\left(\lambda_{1}\right)}{\varphi\left(z_{1}\right)} & \geqslant 1-2 n\left(\frac{e(s+1 / 2)(q-1)}{q^{s+1}}\right)^{2} \\
& \geqslant 1-2 n\left(\frac{e\left(0.5 \log _{q} n+\log _{q} \log _{q} n+1 / 2\right)(q-1)}{q \sqrt{n} \log _{q}^{2} n}\right)^{2} \\
& \geqslant 1-2\left(\frac{e}{\log _{q} n}\right)^{2} .
\end{aligned}
$$

Отсюда вытекают результаты [6]. Если вместо $\varphi\left(z_{1}\right)$ в этом случае использовать $\varphi\left(z_{m}\right)$ при фиксированном $m>1$, то получим еще лучшее приближение. Аналогично можно исследовать поведение $g(q, s, n)$ при $n \rightarrow \infty$ и $s>c \log n$ для любого фиксированного $c>0$.

Для приближения $\varphi\left(\lambda_{1}(q, s)\right)$ можно применять и представление $\lambda_{1}(q, s)$ в виде (7). Аналогично вышеприведенным неравенствам доказывается следующий факт.

Предложение 2. Пусть $q \geqslant 2, s \geqslant 2, m \geqslant 1$ - натуралънъе числа $и$

$$
u(q, m, s)=\sum_{k=1}^{m} \frac{1}{k}\left(\begin{array}{c}
k(s+1)-1 \\
k-1
\end{array}\right)\left(\frac{q-1}{q^{s+1}}\right)^{k}
$$


Тогда

$$
g(q, s, n) \sim q^{n} e^{-n u(q, m, s)} .
$$

$n p u n \rightarrow \infty u$

$$
s \geqslant \frac{1}{m+1} \log _{q} n+\log _{q} \log _{q} n
$$

\section{5. Заключительные замечания}

О представлении $\lambda_{1}(q, s)$ в радикалах. Формула (5) является простой конечной формулой для $\lambda_{1}(q, 2)$. K сожалению, для многих $s$ невозможно выразить $\lambda_{1}(q, s)$ в радикалах. Мы приведем набросок доказательства того, что этого нельзя сделать для $s=5, q=2$.

Нетрудно проверить, что полином $f=x^{5}-x^{4}-x^{3}-x^{2}-x-1$ неразложим по модулю 5 и

$$
f \equiv\left(x^{3}+x^{2}-x+1\right)\left(x^{2}+x-1\right) \quad(\bmod 3) .
$$

Очевидно, многочлены $x^{3}+x^{2}-x+1$ и $x^{2}+x-1$ неразложимы по модулю 3 . Согласно $[1, \S 66]$ группа Галуа полинома $f$, рассматриваемая как группа подстановок его корней, содержит цикл длины пять и транспозицию. Следовательно, она совпадает с симметрической группой степени пять и, в частности, неразрешима.

О разности $g(2, s, n)-b(2, s) \lambda_{1}(2, s)$. Используя то, что не менее половины корней многочлена $A_{q, s}$ лежит в левой полуплоскости (см. §2 и 3 главы XV в [2]), можно убедиться в том, что $\left|g(2, s, n)-b(2, s) \lambda_{1}^{n}\right|<1 / 2$. Таким образом, справедливо следующее уточнение теоремы 1.

Теорема 1'. Пусть $q \geqslant 2, s \geqslant 2$ - натуральные числа, $\lambda_{1}=\lambda_{1}(q, s)$ - наибольший положителъный коренъ многочлена $A_{q, s}(x), a$

$$
b(q, s)=\frac{q\left(\lambda_{1}-1\right)}{(q-1)\left((s+1) \lambda_{1}-s q\right)} .
$$

Тогда при $n \geqslant s$ число $g(q, s, n)$ равно ближайшему $\kappa b(q, s) \lambda_{1}^{n}$ челому числу.

О числах Пизо. Действительное число $a>1$ называется числом Пизо, если оно является корнем такого многочлена $f_{a}(x)$ с целыми коэффициентами и старшим коэффициентом 1 , что модули всех остальных корней $f_{a}(x)$ меньше 1 . В частности, каждое целое $a>1$ (как корень двучлена $x-a$ ) является числом Пизо. Числа Пизо обладают рядом замечательных свойств (см., например, [5, Глава VIII]). Фактически предложение 1 утверждает, что $a=\lambda_{1}(q, s)$ является числом Пизо, где $f_{a}(x)=A_{q, s}(x)$.

О других постановках задач. Теория серий в случайных последовательностях тесно связана с теорией случайных размещений. Эту связь подробно исследовал А. Н. Трунов [10].

Задачу подсчета $g(q, s, n)$ можно формулировать как задачу подсчета слов длины $n$, не содержащих подслов длины $s+1$, состоящих из одинаковых букв. А. А. Евдокимов обратил внимание авторов на то, что в некоторых приложениях требуется оценить число слов длины $n$ с другими множествами запрещенных слов. 
Авторы благодарят Д. Г. Фон-Дер-Флаасса за ценные советы, а также М. В. Фокина за замечание о числах Пизо.

\section{Список литературы}

1. Ван дер Варден Б. Л. Алгебра. Наука, Москва, 1976.

2. Гантмахер Ф. Р. Теория матрич. Гостехиздат, Москва, 1953.

3. Гельфонд А. О. Исиисление конечных разностей. Наука, Москва, 1967.

4. Гончаров В. Л. Из области комбинаторики. Известия АН СССР, Серия матем. (1944) 8, №1, 3-48.

5. Касселс Дж. В. С. Введение в теорию диофантовых приближений. ИЛ, Москва, 1961.

6. Коршунов А. Д. О числе бинарных слов с заданной длиной максимальной серии, I. Дискретный анализ и исследование операчий (1997) 4, №4. 13-46.

7. Курош А. Г. Курс высшей алгебры. Наука, Москва, 1968.

8. Маркушевич А. И. Возвратные последовательности. Наука, Москва, 1975.

9. Савельев Л. Я. Серии в марковских последовательностях. Сибирский матем. ж. (1991) 32, №4, 116-132.

10. Трунов А. Н. Предельные теоремы в задаче о размещении одинаковых частиц по различным ячейкам, Труды МИАН СССР им. В. А. Стеклова (1986) 177, 147-164.

11. Уиттекер Э. Т., Ватсон Дж. Н. Курс современного анализа, Ч. 1. ГИФМЛ, Москва, 1962.

12. Фихтенгольц Г. М. Курс дифференциального $и$ интегрального исчисления, т. II. ГИФМЛ, Москва, 1962.

13. Холл М. Комбинаторика. Мир, Москва, 1970.

14. Savelyev L. Ya, Runs in finite Markov chains. Probabilistic Methods in Discrete Mathematics. Proc. 3rd International Petrozavodsk Conf. VSP, Utrecht, 1993, pp. 437-450.

Статья поступила 04.02.1998. 